\title{
Melatonin Treatment of Winter Depression Following Total Sleep Deprivation: Waking EEG and Mood Correlates
}

\author{
Konstantin V Danilenko*, and Arcady A Putilov ${ }^{2}$ \\ 'Institute of Internal Medicine, Siberian Branch of the Russian Academy of Medical Sciences, Novosibirsk, Russia; ${ }^{2}$ Research Institute for \\ Molecular Biology and Biophysics, Siberian Branch of the Russian Academy of Medical Sciences, Novosibirsk, Russia
}

\begin{abstract}
Patients with winter depression (seasonal affective disorder (SAD)) commonly complain of sleepiness. Sleepiness can be objectively measured by spectral analysis of the waking electroencephalogram (EEG) in the $1-10 \mathrm{~Hz}$ band. The waking EEG was measured every $3 \mathrm{~h}$ in 16 female SAD patients and 13 age-matched control women throughout a total sleep deprivation of $30 \mathrm{~h}$. Melatonin (or placebo) under double-blind conditions was administered subsequently $(0.5 \mathrm{mg}$ at $1700 \mathrm{~h}$ for 6 days), appropriately timed to phase advance circadian rhythms, followed by reassessment in the laboratory for $12 \mathrm{~h}$. The increase in EEG power density in a narrow theta band (5$5.99 \mathrm{~Hz}$, derivation $\mathrm{Fz}-\mathrm{Cz}$ ) during the $30 \mathrm{~h}$ protocol was significantly attenuated in patients compared with controls (difference between linear trends $p=0.037)$. Sleepiness $(p=0.092)$ and energy $(p=0.045)$ self-ratings followed a similar pattern. Six patients improved after sleep deprivation ( $\geqslant 50 \%$ reduction on $\mathrm{SIGH}_{-} \mathrm{SAD}_{22}$ score). EEG power density dynamics was correlated with clinical response to sleep deprivation: the steeper the build-up (as in controls), the better the improvement $(p<0.05)$. There was no differential effect of melatonin or placebo on any measure; both treatments stabilized the improvement. Overall, patients with winter depression manifest similar wake EEG characteristics as long sleepers or late chronotype with respect to an insufficient build-up of homeostatic sleep pressure. Sleep deprivation was an effective antidepressant treatment for some patients, but evening melatonin was not more efficacious than placebo in sustaining this antidepressant effect.

Neuropsychopharmacology (2005) 30, 1345-1352, advance online publication, 16 February 2005; doi: 10.1038/sj.npp. 1300698
\end{abstract}

Keywords: winter depression (SAD); sleep deprivation; melatonin; wake EEG; spectral analysis; sleepiness

\section{INTRODUCTION}

Research into winter depression (seasonal affective disorder (SAD), winter type) has an unique history. Unlike other affective disorders, it was discovered by investigations in the field of chronobiology (Lewy et al, 1982; Rosenthal et al, 1984) rather than through medical practice. Since the first description of winter depression (Rosenthal et al, 1984), a number of hypotheses have been proposed to explain its underlying pathophysiology and its successful treatment by bright light. However, none of these hypotheses is fully compatible with existing empirical results (see the review by Lam and Levitan, 2000).

Winter depression is characterized by so-called atypical depressive symptoms which include hypersomnia, difficulty waking up in the morning, and daytime sleepiness. These abnormal features disappear, or even the opposite symptom

*Correspondence: Dr KV Danilenko, Institute of Internal Medicine SB RAMS, Bogatkova 175/I, Novosibirsk 630089, Russia, Tel: + 73832 679755, Fax: + 73832 642516, E-mail: dani@irs.ru

Received 30 September 2004; revised 28 December 2004; accepted 7 January 2005

Online publication: 17 January 2005 at http://www.acnp.org/citations/ Npp0 I 1 705040463/default.pdf of hyposomnia can appear throughout summer remission (Rosenthal et al, 1984). Sleep in SAD patients is remarkably normal (Anderson et al, 1994; Brunner et al, 1996; Palchikov et al, 1997, Koorengevel et al, 2002a) and has little resemblance to sleep in other major affective disorders (eg Benca et al, 1992). Sleep electroencephalographic (EEG) studies have failed to support clinical and epidemiological evidence that describes changes in subjective sleep quality with season or after bright light treatment (summarized in Koorengevel et al, 2002a).

Studies on sleep regulation have become more specific due to two methods: first, sleep-EEG power density in deltatheta frequencies correlates with depth of recovery from sleep need (Borbély et al, 1981; Dijk et al, 1987), and second, waking-EEG power density in theta-alpha frequencies correlates with increasing sleep need during prolonged wakefulness (Åkerstedt and Gillberg, 1990; Cajochen et al, 1995). A close correlate of the pronounced increasing trend of subjective sleepiness are the low frequency bands between 1 and $10 \mathrm{~Hz}$ in the wake-EEG (Cajochen et al, 1995, 1998; Aeschbach et al, 1999; Dumont et al, 1999), particularly when the electrodes are placed at frontal rather than more posterior areas of the scalp (Cajochen et al, 1999, 2002; Finelli et al, 2000). 
In application to SAD research, this methodology was explored for the first time by Cajochen et al (2000) in a controlled constant routine protocol of $40 \mathrm{~h}$ wakefulness. Although the EEG was recorded only four times, the results revealed that the typical build-up of power density in the course of extended wakefulness was attenuated in winter depressive compared to healthy women. The time course of EEG power density in the theta range $(6-9 \mathrm{~Hz})$ was paralleled by similar changes in the time course of subjective sleepiness rated every $30 \mathrm{~min}$ : the linear trend was significantly less prominent in patients compared to controls (Cajochen et al, 2000). As the sample sizes were small and the EEG sampling interval was long, these findings require replication and extension.

In addition to allowing controlled investigation of circadian rhythms and increasing sleep propensity, the constant routine protocol of $40 \mathrm{~h}$ extended wakefulness is a total sleep deprivation. As in other groups of major depression (reviewed by Wirz-Justice and Van den Hoofdakker, 1999), total sleep deprivation in SAD can also induce a rapid antidepressant response (Graw et al, 1998). The improvement after sleep deprivation is usually shortlasting, with relapse after recovery sleep (Wirz-Justice and Van den Hoofdakker, 1999). The depressive relapse has been prevented by subsequent 1 week morning light therapy in non-SAD (Neumeister et al, 1996; Bloching et al, 2000; Loving et al, 2002). Riemann et al (1999) applied a sleep advance schedule in patients with melancholic depression and found that an advance of sleep time during 1 week was a more effective stabilizer of the antidepressant effect of sleep deprivation than a delay of sleep.

SAD patients tend to be phase delayed in their circadian rhythms (Wirz-Justice et al, 1995), especially if hypersomnic (Putilov et al, 1996; Avery et al, 1997). No other circadian rhythm abnormalities have been consistently found in winter depressives (summarized in Koorengevel et al, 2002b). With respect to this tendency to phase delay, a phase advance appears to be part of the mechanism mediating the antidepressant response to early morning bright light (Lewy et al, 1987; Terman et al, 2001).

Since not only morning light induces a phase advance of the circadian system, but also late afternoon melatonin administration (Lewy et al, 1998a), the phase shift hypothesis of SAD pathophysiology would suggest an antidepressant effect of afternoon treatment of SAD patients with melatonin (Lewy et al, 1987). Indeed, a small pilot study found mood-elevating effects of afternoon melatonin (Lewy et al, 1998b), but this was not replicated in the larger controlled trial (Lewy et al, 2003).

The above considerations led to our planning a combined sleep deprivation and melatonin administration treatment study of SAD. If afternoon melatonin has antidepressant potential due to an advance shift of circadian phase, it might be taken to prevent relapse after sleep deprivation, rather than using the more difficult sleep phase advance procedure (Riemann et al, 1999). In addition, using melatonin instead of bright light provides a straightforward possibility of blind comparison with placebo. In addition to clinical ratings, we calculated the time course of waking EEG power density in winter depressives and in healthy controls. We hypothesized that the normal build-up of sleep pressure during prolonged wakefulness would be attenuated in SAD and that after evening melatonin administration EEG parameters would approach normal values.

\section{METHODS}

\section{Participants}

The study was carried out between December 1999 and February 2000 at the Research Institute for Molecular Biology and Biophysics in Novosibirsk $\left(55^{\circ} \mathrm{N}\right.$, with sunrise at this time of the year between 0800 and $0900 \mathrm{~h}$ ). SAD patients and control subjects were recruited via advertisements in local newspapers and mass media or via previous patients' database. They were unmedicated, in good general health, free of major sleep disorders, and were not engaged in shift work or long-distance travel in the prior 2 months.

In all, 16 DSM-IV-diagnosed depressive SAD women (American Psychiatric Association, 1994) and 17 agematched healthy women participated in the study. All patients manifested hypersomnia as defined in the interview, with at least $1 \mathrm{~h}$ more sleep in winter than during their euthymic state in summer (Avery et al, 1997). Four of the control subjects were later excluded from the analyses because their questionnaire data revealed mild seasonality of mood and well-being, that is, considerable wintersummer difference in sleep need (1-3h) and a borderline score on the Seasonal Pattern Assessment Questionnaire (Rosenthal et al, 1987). There was no significant age difference between the patient (18-54 year) and control (21-48 year) groups (mean \pm SEM: $35.1 \pm 2.6$ vs $29.2 \pm 2.6$ years, $p=0.13$ ).

The majority of the study participants had no strict working hours. The habitual nocturnal sleep times of the subjects ranged from 2200 to $0230 \mathrm{~h}$ for going to bed and from 0615 to $1100 \mathrm{~h}$ for waking up. For 5-7 days prior to the study, they were asked to maintain their own regular habitual bedtime within the range of $\pm 1 \mathrm{~h}$. They were also asked to keep daily sleep logs during 5 pre-treatment days and in the course of the treatment week, and these were checked for compliance. The study entry dates were not controlled for menstrual cycle phase.

\section{Experimental Protocol}

Subjects were admitted to a research unit of the Institute in groups of $4-6$. They arrived at $0900-0930 \mathrm{~h}$ on Day 1 and left the laboratory shortly after $1600 \mathrm{~h}$ on Day 2 . The $30 \mathrm{~h}$ experimental procedure included clinical assessment and 11 EEG recordings and subjective ratings at 3 -h intervals starting from $1000 \mathrm{~h}$. Ambient light intensity in the rooms was $\sim 100$ lux at the angle of gaze. Subjects were not allowed to walk outside the laboratory facilities during the daytime, but could go briefly outdoors at night. The study personnel ensured that they always remained awake.

For 6 days, starting from Day 2, both patients and controls were randomly assigned to take sublingually at $1700 \mathrm{~h}$ either placebo or $0.5 \mathrm{mg}$ of melatonin (as powder prepared from capsules of $3 \mathrm{mg}$, California Health ${ }^{\circledR}$, USA) at home. This dose was chosen as being in the physiological range (reviewed by Zhdanova and Wurtman, 1997) as well as having been shown to successfully phase advance circadian rhythms in winter depressives (Lewy et al, 
2003). On Day 8, the subjects returned to the research unit to repeat the first $12 \mathrm{~h}$ of the experimental procedure (i.e. four EEG recordings and subjective ratings between 1000 and $1900 \mathrm{~h}$ ).

The experimental protocol was approved by the Ethics Committee of the Siberian Branch of the Russian Academy of Medical Sciences and all study participants gave written informed consent.

\section{Measurements}

Sleep duration and mid-sleep time were calculated from sleep logs covering the 5-day pre-treatment and 6-day treatment intervals. The waking EEG was recorded from two derivations: $\mathrm{Fz}-\mathrm{Cz}$ (active electrode placed in the middle from the top of the head to forehead) and C4-A1 (active electrode placed at $1 / 3$ distance from the top of the head to the right ear). Ten 20 conductive paste (Nicolet Biomedical, USA) was used to fix the electrodes that were removed after each recording (their exact position was preliminarily inked by a permanent marker). For each 3-4 min EEG recording, the subject sat in a comfortable chair. She was instructed to relax and fixate her eyes on a spot on the wall. EEG signals were sampled and computerized via a multi-channel recorder of physiological signals BI-01R (produced by the Research Institute for Molecular Biology and Biophysics, Novosibirsk, Russia). Waking EEG power spectra of 4-s epochs were calculated for each $1-\mathrm{Hz}$ frequency band from 1 to $20 \mathrm{~Hz}$. All 4-s epochs confounded by artifacts (due to eye blinking, movements, slow rolling eyes, etc.) were well defined by excessive power density values, identified by visual inspection of the EEG signal, and eliminated from the analysis. At each time of electrode placement for EEG, participants selfrated their sleepiness on the Karolinska Sleepiness Scale (Gillberg et al, 1994) and mood and energy levels on two Visual Analog Scales.

The 29-item Structured Interview Guide for the Hamilton Depression Rating Scale-Seasonal Affective Disorder Version (SIGH-SAD; Williams et al, 1988)-was completed between 1200 and $1500 \mathrm{~h}$ three times over the study period. An initial SIGH-SAD score was obtained on Day 1. After the sleep deprivation night (Day 2), seven of 29 items were not included, because they could not be rated adequately either due to the short interval between the ratings (H5 itemweight loss and A2 item - weight gain) or due to absence of sleep (H6-8 items - insomnia in the early, middle, and late night, A6 item-hypersomnia, H18 item-feeling worse before or after sleep). This shortened version of SIGH-SAD is henceforth described as SIGH-SAD 22 . The final SIGHSAD score was obtained on Day 8 when the subjects were restudied after having been treated with either melatonin or placebo.

\section{Statistical Analyses}

Data were subjected to analysis of variance for repeated measures (rANOVA); Huynh-Feldt's corrected probabilities $(p)$ are reported for particular Fisher's coefficients. Unpaired $t$-tests were applied to locate significant differences between two values. Sometimes, the curves were represented as deviation from the mean or $z$-transformed to account for inter-individual differences in the mean values during the unpaired $t$-tests or for the aim of crosscorrelation.

\section{RESULTS}

\section{Baseline}

In spite of complaints of hypersomnia, sleep diaries did not show that SAD patients slept significantly longer than controls during four nights preceding the baseline night (Figure 1: pooled data from Day -5 to Day -2 ). Sleep duration was rather long in both patients and controls, mean \pm SEM: $9.02 \pm 0.23$ vs $8.56 \pm 0.29 \mathrm{~h}(p=0.21)$, perhaps related to the fact that the majority did not go out early to work. Nonetheless, patients woke up significantly later than controls: $9.06 \pm 0.28$ vs $8.23 \pm 0.28 \mathrm{~h}(p=0.047)$. The difference in bedtime or mid-sleep time did not reach a significance threshold $(24.18 \pm 0.41$ vs $23.67 \pm 0.17 \mathrm{~h}$, $p=0.29 ; 4.62 \pm 0.33$ vs $3.95 \pm 0.18 \mathrm{~h}, p=0.11$, respectively).

Both groups slept significantly less on the last baseline night (Day -1$)$ compared to the previous 4 days $(p<0.0001$ for patients, $p=0.012$ for controls; Figure 1) because study participants needed to wake up earlier to reach the laboratory in time for admission to the trial. However, the difference in sleep loss between patients and controls was not significant $(1.60 \pm 0.22$ vs $0.91 \pm 0.31 \mathrm{~h}, p=0.073)$.

\section{Days 1-2 (Sleep Deprivation)}

Waking EEG power density varied considerably across individuals: from two- to six-fold inter-individual differences were obtained for different frequency bands. These differences resulted in rather big SEMs in both patient and control groups (column 'Group mean' of Table 1 and Figure 2).

The variations in EEG power density over the $30 \mathrm{~h}$ episode of sustained wakefulness were significant in nearly half of the frequency bands in both patients and controls (column 'Time course' of Table 1). The significant difference in the time course of EEG power density between patients and controls was revealed in only one frequency band,

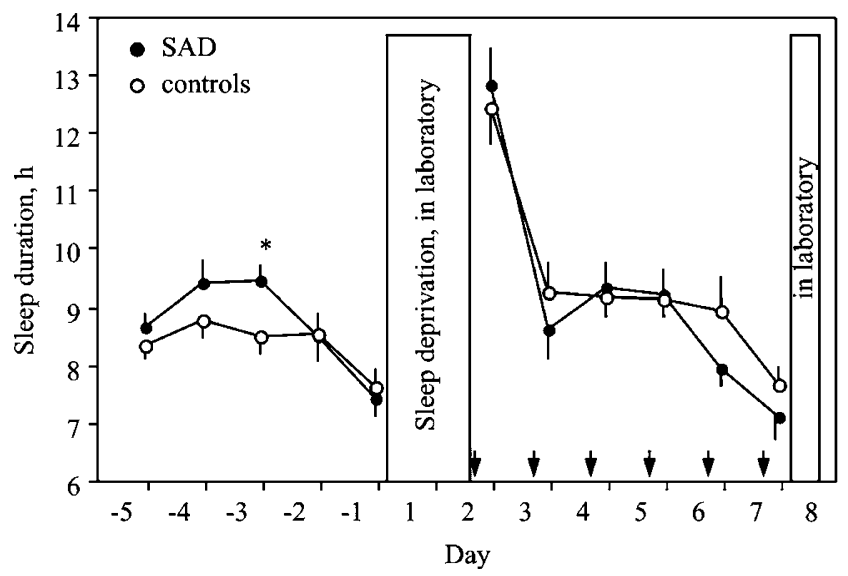

Figure I Sleep duration (mean \pm SEM) derived from sleep logs during baseline days $(-5$ to -1$)$ and experimental days (2-7). Arrows indicate time of melatonin (or placebo) intake. $* p<0.05$. 
Table I Waking EEG Power Density (Derivation Fz-Cz) and Subjective Self-Ratings Over 30 h Wakefulness in 16 SAD Patients and I3 Healthy Controls

\begin{tabular}{|c|c|c|c|c|c|c|c|}
\hline & \multicolumn{2}{|c|}{ Group mean \pm SEM } & \multicolumn{2}{|c|}{ Time course, $p$} & \multirow{2}{*}{$\begin{array}{l}\text { Group } \times \text { Time } \\
\text { interaction, } p\end{array}$} & \multicolumn{2}{|c|}{ Linear trend, $p$} \\
\hline & Patients & Controls & Patients & Controls & & Patients & Controls \\
\hline \multicolumn{8}{|c|}{ Waking EEG power density, $\mu V^{2}$} \\
\hline $\mathrm{I}-1.99, \mathrm{~Hz}$ & $113.4 \pm 3.6$ & $115.4 \pm 4.4$ & 0.023 & 0.092 & 0.099 & 0.020 & $0.04 I$ \\
\hline $4-4.99$ & $96.5 \pm 4.1$ & $100.8 \pm 4.6$ & 0.103 & 0.0003 & 0.28 & 0.0010 & 0.040 \\
\hline $5-5.99$ & $92.5 \pm 4.5$ & $94.3 \pm 5.1$ & 0.59 & 0.0001 & 0.042 & 0.13 & 0.0015 \\
\hline $6-6.99$ & $94.3 \pm 4.7$ & $96.0 \pm 6.0$ & 0.37 & 0.020 & 0.15 & 0.18 & 0.0013 \\
\hline $7-7.99$ & $95.9 \pm 5.6$ & $103.4 \pm 8.4$ & 0.091 & 0.12 & 0.58 & 0.078 & 0.0012 \\
\hline $8-8.99$ & $100.1 \pm 8.8$ & $|10.8 \pm| 1.1$ & 0.28 & 0.021 & 0.47 & 0.16 & 0.042 \\
\hline $12-12.99$ & $77.7 \pm 5.6$ & $85.5 \pm 8.9$ & 0.24 & 0.0056 & 0.48 & 0.16 & 0.59 \\
\hline $13-13.99$ & $69.2 \pm 4.6$ & $66.2 \pm 4.9$ & 0.059 & 0.0017 & 0.27 & 0.12 & 0.56 \\
\hline $14-14.99$ & $67.0 \pm 4.6$ & $61.6 \pm 4.2$ & 0.015 & 0.0089 & 0.73 & 0.20 & 0.91 \\
\hline $15-15.99$ & $67.3 \pm 5.1$ & $59.9 \pm 3.9$ & 0.038 & 0.084 & 0.46 & 0.20 & 0.57 \\
\hline $16-16.99$ & $67.7 \pm 5.5$ & $59.7 \pm 3.8$ & 0.0014 & 0.105 & 0.35 & 0.38 & 0.78 \\
\hline $17-17.99$ & $66.6 \pm 5.3$ & $60.1 \pm 3.8$ & 0.0075 & 0.082 & 0.71 & 0.16 & 0.69 \\
\hline $18-18.99$ & $64.2 \pm 5.0$ & $60.1 \pm 3.7$ & 0.034 & 0.029 & 0.58 & 0.069 & 0.41 \\
\hline $19-19.99$ & $64.1 \pm 4.2$ & $60.8 \pm 3.6$ & 0.0004 & 0.058 & 0.36 & 0.0058 & 0.13 \\
\hline \multicolumn{8}{|c|}{ Subjective self-ratings, score } \\
\hline
\end{tabular}

*** $p<0.01$, ***** $p<0.001$ compared to controls.

Bold represents significant $p$-values.

$5-5.99 \mathrm{~Hz}$, and in one derivation, $\mathrm{Fz}-\mathrm{Cz}$ (column 'Group $\times$ Time interaction' of Table 1). However, none of the particular time point values were significantly different between patients and controls as indicated by unpaired $t$-tests (Figure 2). Significant differences emerged for the relative values: when the individual curves were represented as deviation from the mean (referred to as $100 \%$ ), power density was higher in patients than controls at 1000 on Day $1(98.2 \pm 2.2 v s 90.6 \pm 2.7 \%, p=0.035)$ and lower at 1600 on Day $2(102.3 \pm 1.5$ vs $113.7 \pm 2.5 \%, p=0.0004)$, with the rANOVA group interaction $p=0.033$.

In the control group, power density increased progressively, and a highly significant linear trend was found for all frequency bands between 5 and $8 \mathrm{~Hz}$ (theta waves). In contrast, the linear trend was nonsignificant in these frequency bands in the SAD patient group (column 'Linear trend' of Table 1). The mean individual slope of the linear trend for the band $5-5.99 \mathrm{~Hz}$ was significantly steeper in controls than in patients, $1.47 \pm 0.37$ vs $0.27 \pm 0.38$ $(p=0.037)$. The results obtained for derivation $\mathrm{C} 4-\mathrm{A} 1$ were very similar to those for derivation $\mathrm{Fz}-\mathrm{Cz}$, but the statistical output of the main interest-Group $\times$ Time interaction-did not attain the significance threshold.

SAD patients rated their levels of sleepiness higher and energy and mood levels lower than controls (column 'Group mean' of the lower part of Table 1 and Figure 2). Similar to the results for EEG power density at $5-5.99 \mathrm{~Hz}$, rANOVA yielded a significant interaction, revealing the differences between patients and controls in the time course of sleepiness (column 'Group $\times$ Time interaction' of the lower part of Table 1). This difference again reflected the presence of a generally increasing sleepiness trend in the control group and nonsignificance in the patient group (column 'Linear trend' of the lower part of Table 1). Averaged over individuals, the trends tended to be steeper in controls than in patients, $0.24 \pm 0.03$ vs $0.14 \pm 0.04 \quad(p=0.092)$. The dynamics of energy self-ratings mirrored the dynamics of subjective sleepiness (lower part of Table 1; not shown in the figure). The mean linear decreasing trend was significantly steeper in the control group compared to the patient group: $-0.27 \pm 0.04 v s-0.12 \pm 0.06(p=0.045)$. Even though SAD patients had overall lower mood levels than 


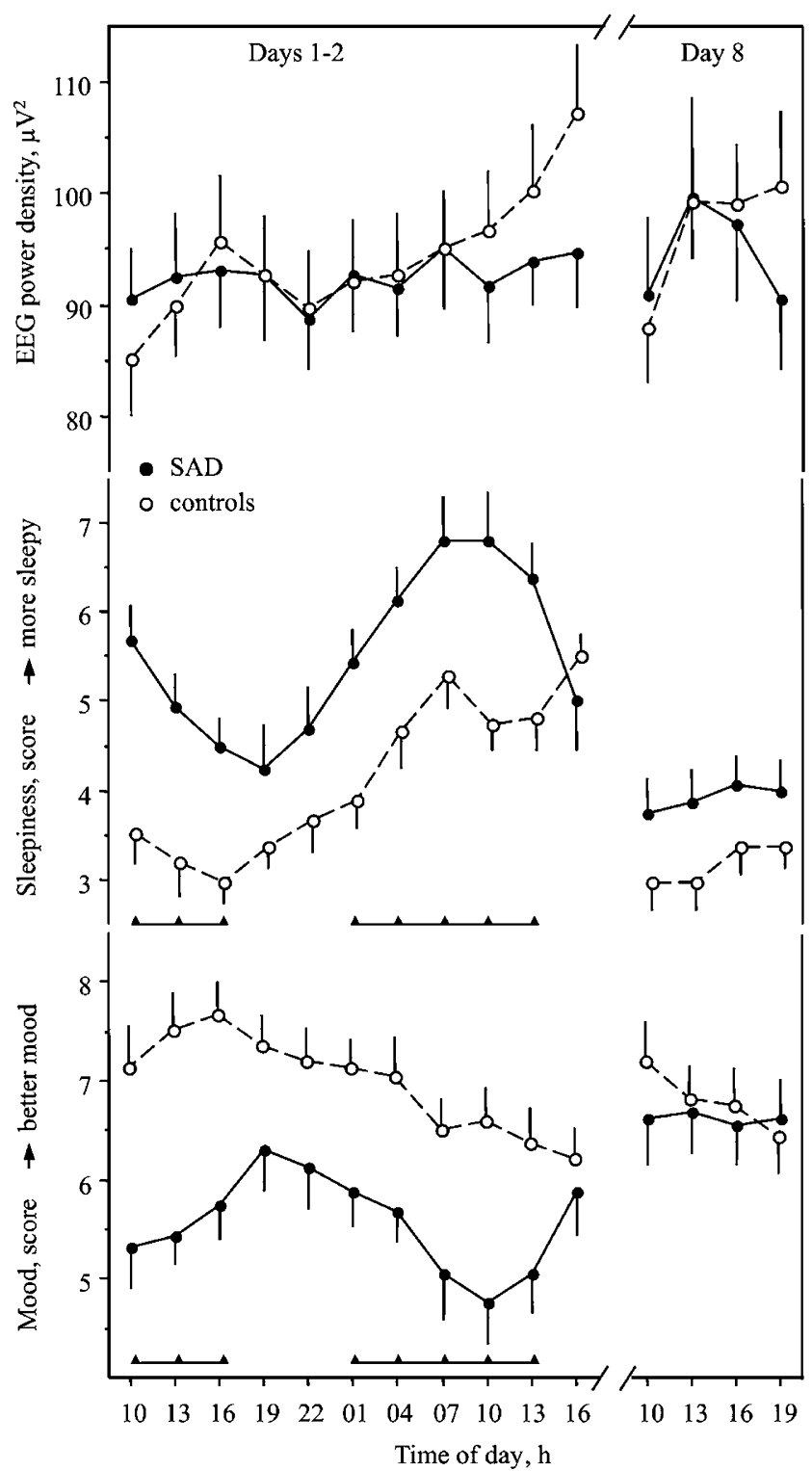

Figure 2 Dynamics of waking EEG power density (at $5-5.99 \mathrm{~Hz}$, derivation $\mathrm{Fz}-\mathrm{Cz}$ ), subjective sleepiness (Karolinska Sleepiness Scale), and mood (Visual Analog Scale) (mean values \pm SEM) in 16 SAD patients and 13 controls during $30 \mathrm{~h}$ of continuous wakefulness (Days I-2) and during 12 daytime hours after 6 days of melatonin (or placebo) intake (Day 8). Triangles beneath the corresponding curves indicate significant difference between SAD and controls (at least $p<0.05$, unpaired $t$-tests).

controls, mood dynamics over $30 \mathrm{~h}$ protocol did not reveal a significant interaction term (lower part of Table 1; Figure 2), and the difference in the linear trends was nonsignificant $(p=0.17)$. Mood in controls declined with time awake (Table 1; Figure 2). In contrast, SAD patients showed a clear-cut circadian rhythm of subjective mood (Figure 2).

There was no significant correlation between individual means of subjective sleepiness and EEG power density. However, a significant cross-correlation was found between the dynamics of EEG power density (\% deviation from the mean at $5-5.99 \mathrm{~Hz}$ ) and subjective sleepiness (standard, or $z$-score) in the control group $(p<0.0001, N=13)$; in the patient group it was nonsignificant $(p=0.98, N=16)$.

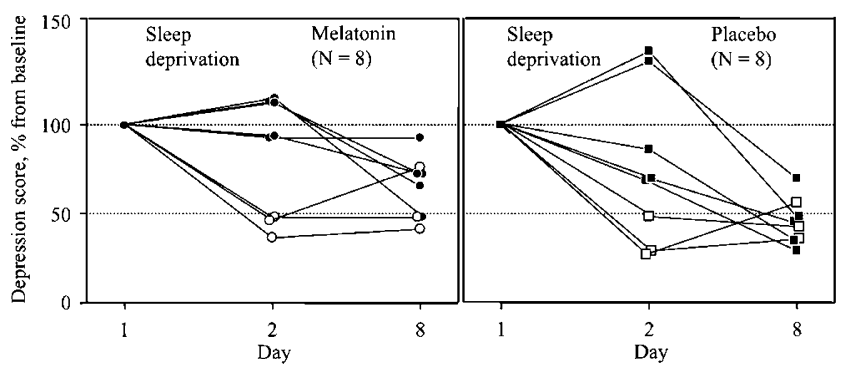

Figure 3 Individual depression ratings in 16 SAD patients after total sleep deprivation (Day 2) and following melatonin ( $0.5 \mathrm{mg}$ sublingual at 1700, left panel) or placebo intake (right panel) for 6 days (Day 8). Depression ratings were based on SIGH-SAD's 22 selective items (see Methods), and the initial score (at Day I) was referred to as 100\%. Open symbols represent responders to sleep deprivation ( $\geqslant 50 \%$ improvement).

The effect of sleep deprivation on depression ratings was analyzed only in the patient group $(N=16)$. Overall, the SIGH-SAD 22 score tended to decrease after the sleep deprivation night from $29.6 \pm 1.9$ to $23.6 \pm 3.4(p=0.054)$. A reduction of at least $50 \%$ was found in six of 16 patients (Figure 3). Similar results were obtained using the $\mathrm{HDRS}_{17}$ subscale of SIGH-SAD (cf. description of $\mathrm{HDRS}_{17}$ in Graw et al, 1998).

The \% change in depression score significantly correlated with the EEG power density slope (for SIGH-SAD $22 r=0.59$, $p=0.014$; for $\mathrm{HDRS}_{17} r=0.69, p=0.0023$ ). This correlation indicates that, over the $30 \mathrm{~h}$ sleep deprivation episode, patients with a steeper trend in EEG power density (similar to normal sleepers) were more likely to improve mood after sleep deprivation than patients with a flatter slope of EEG power density rise.

\section{Day 8 (Post-Treatment)}

After the 1-day sleep deprivation, all participants received melatonin (eight patients, five controls) or placebo (eight patients, eight controls) at 1700 for 6 days. An acute soporific effect within 1-2 h after pill intake was reported in interview by four patients and two controls of the 13 subjects taking melatonin, and by one patient and one control of the 16 subjects taking placebo $\left(p=0.092, \chi^{2}\right.$-test; $p=0.04$, Mann-Whitney test).

No influence of melatonin intake on the time course or mean value of daytime EEG power density was noted as revealed by rANOVAs, neither was there a significant effect of treatment (melatonin vs placebo) on the daytime course of subjective ratings.

Those subjects who were blindly assigned to melatonin did not show any advance of their mid-sleep time compared to the pre-treatment condition $(3.37 \pm 0.28 v s 3.69 \pm 0.33 \mathrm{~h})$. Their treatment mid-sleep time was found to be close to that of subjects assigned to placebo $(3.89 \pm 0.17 v s 3.74 \pm 0.17 \mathrm{~h})$.

Changes in the depression SIGH-SAD 22 score in patients are depicted in Figure 3. No differences in scores between melatonin $(N=8)$ and placebo $(N=8)$ subgroups were found, either at baseline before sleep deprivation, on Day $1(29.6 \pm 3.1 v s 29.6 \pm 2.4)$, or after it on Day $2(24.5 \pm 4.6$ vs 22.6 \pm 5.3$)$. After both melatonin and placebo treatment, the scores were nonsignificantly decreased further by 
$14.0 \pm 13.3$ and $27.2 \pm 16.5 \%$, respectively. These two decrements did not differ, as indicated by unpaired $t$-tests $(p=0.54)$. Even when those patients who positively responded to sleep deprivation on Day $2(N=3$ in each subgroup) were excluded from the analysis, a significant difference between these two decrements did not emerge $(33.0 \pm 9.5$ vs $53.8 \pm 6.2 \%, p=0.103)$. Instead, a regression towards the mean was observed: higher depression score on Day 2 predicted better response on Day $8 \quad(r=0.74$, $p=0.012, N=10)$.

Subjective ratings confirmed the positive depression dynamics: mood and energy increased and sleepiness decreased on Day 8 compared to Day 1 (Figure 2; $p<0.05)$, whereas no significant changes in the subjective ratings were found in controls. On Day 8, there was no significant effect of factors Group (SAD vs controls), Treatment (melatonin vs placebo), or their interaction on the time course (four time points 1000, 1300, 1600, 1900) of subjective ratings and EEG power density (Figure 2). Thus, sleepiness, energy, and mood on Day 8 were 'normalized' in SAD patients.

\section{DISCUSSION}

\section{Waking EEG Throughout $30 \mathrm{~h}$ Wakefulness}

The results of the present study suggest that SAD patients differ from non-depressed controls with respect to the dynamics of EEG power density during $30 \mathrm{~h}$ of sustained wakefulness. The statistical difference was found in the range of theta waves, frequency band $5-5.99 \mathrm{~Hz}$, in the fronto-central derivation, $\mathrm{Fz}-\mathrm{Cz}$. The differences in sleepiness and energy self-ratings followed a similar pattern. Our results are in line with earlier findings reported by Cajochen et al (2000).

Controls exhibited the well-known increasing trend in the theta-alpha activity range. This trend is considered to reflect the so-called wake-dependent, or homeostatic component of sleep regulation (Cajochen et al, 1995, 1999; Aeschbach et al, 1999; Dumont et al, 1999), which is most prominent when recorded in the fronto-central derivation Fz-Cz (Cajochen et al, 1999, 2002; Finelli et al, 2000) and which is closely associated with subjective sleepiness (Cajochen et al, 1998; Aeschbach et al, 1999; Dumont et al, 1999).

The patients' data were not so consistent: there was no significant increasing trend in EEG power density, and the association of EEG power density and sleepiness was poor. The lack of association may be related to the overall prevalent sleepiness, which is a characteristic symptom of SAD. The existing sleepiness could have attenuated any build-up of the waking EEG power density - the latter was always high. SAD patients appear to retain this attenuated build-up of waking EEG power density even after successful light therapy in winter as well as in summer, both conditions when they are not depressed and not hypersomnic (Cajochen et al, 2000). This suggests a trait and not state dependency. The insufficiency of build-up is similar to the slow build-up in long sleepers (Aeschbach et al, 2001), or in evening chronotypes (Taillard et al, 2003). Since SAD patients (at least subjectively) consider themselves long sleepers, and appear to be more often late chronotypes
(Elmore et al, 1993; Putilov et al, 2000; Graw et al, unpublished data), the present finding may be related to these physiological characteristics and not their winter depressive diagnosis per se.

In contrast to the homeostatic component, the circadian component of the EEG power density dynamics is similar in SAD patients and controls (as also shown by Cajochen et al, 2000), with a characteristic drop around 2200 in both groups (Figure 2) that is known as the evening 'sleepforbidden zone' or 'wake-maintenance zone' (Lavie, 1986; Strogatz et al, 1987).

\section{Treatment Effects}

At least $50 \%$ reduction of depression score after sleep deprivation was shown in $38 \%$ of the SAD patients, of the same order as the $52 \%$ reported by Graw et al (1998). An interesting correlation between the change in depression score and the EEG power density build-up suggests that EEG power density may rapidly change in parallel with mood during sleep deprivation. Another explanation could be that hypersomnic winter depressives have problems staying awake during sleep deprivation, thus having microsleep episodes that neither they nor the study personnel register (Hemmeter et al, 1998). The more the microsleeps, the less the patients improve (Hemmeter et al, 1998). Such an unrecognized dissipation of sleep pressure may result in a flatter build-up of EEG power density and, thus, one explanation for the correlation we found in our study may be the inverse link between sleep pressure dissipation and mood.

After sleep deprivation, the follow-up adjunct treatment led to a stabilization of the antidepressant effect of sleep deprivation, without any greater effectiveness of melatonin than placebo. This is suggestive of a large placebo effect, emphasizing the importance of patients' therapeutic hopes and their tendency to respond positively to any procedure of investigational treatment. The patients had the possibility of verbalizing their problems and discussing their symptoms with physicians, research assistants, and other participating subjects, even though blind to treatment. In addition, the unusual form (powder) and method of intake (sublingual) of the dispensed substances might also have contributed to the high placebo response rate. The rate of placebo response could not be determined adequately in such a combination treatment trial, but was probably higher than the $\sim 30 \%$ usually seen after 1 week placebo treatment of winter depression (eg Lam et al, 1995; Eastman et al, 1998).

According to the sleep logs, there was no advance of sleep timing in either SAD patients or controls after melatonin. The sleep-wake cycle is not a good marker of the biological clock, since it could have been masked or maintained in a regular phase position by the influence of other $24 \mathrm{~h}$ time cues that were left unchanged throughout the treatment week. Although not measured, melatonin may have indeed advanced 'internal time' (ie its own and other core circadian rhythms) as it is known to do so when administered in the early evening (Zaidan et al, 1994; Lewy et al, 1998a). Thus, we did not estimate the phase-resetting effects of melatonin (that theoretically would have been of the order of $1 \mathrm{~h}$ ), but we did obtain, under realistic clinical conditions, 
no evidence that melatonin is an antidepressant in patients with SAD (in contrast to our positive experience with bright light in similar populations (Palchikov et al, 1997)). Melatonin treatment also did not affect the dynamics of EEG power density and subjective ratings more than placebo.

In conclusion, our main result replicates and extends in detail across $30 \mathrm{~h}$ prolonged wakefulness the previous finding that patients with winter depression have a slower increase of sleep pressure compared to nondepressed controls (Cajochen et al, 2000). This result is based on spectral analysis of the waking EEG and subjective sleepiness. Our study also suggests that subsequent evening administration of melatonin was not more effective than placebo to maintain the therapeutic effect of sleep deprivation.

\section{ACKNOWLEDGEMENTS}

This study was supported by the 'Russian Fund' of the Society for Light Therapy and Biological Rhythms (SLTBR). We thank Olga Donskaya for assistance in EEG recordings, Olga Jafarova for technical consulting on analysis of EEG power density with BI-01R, and Olga Sokorova for the work with statistical files. We are very grateful to Drs Anna WirzJustice and Christian Cajochen for reading, commenting and English editing earlier versions of the manuscript, and the anonymous reviewers for their helpful comments.

\section{REFERENCES}

Aeschbach D, Matthews JR, Postolache TT, Jackson MA, Gieses HA, Wehr TA (1999). Two circadian rhythms in the human electroencephalogram during wakefulness. Am J Physiol 277: R1771-R1779.

Aeschbach D, Postolache TT, Sher L, Matthews JR, Jackson MA, Wehr TA (2001). Evidence from the waking electroencephalogram that short sleepers live under higher homeostatic sleep pressure than long sleepers. Neuroscience 102: 493-502.

Åkerstedt T, Gillberg M (1990). Subjective and objective sleepiness in the active individual. Int J Neurosci 52: 29-37.

American Psychiatric Association (1994). Diagnostic and Statistical Manual of Mental Disorders, 4th edn. American Psychiatric Press: Washington, DC.

Anderson JL, Rosen LN, Mendelson WB, Jacobson FM, Skwerer RG, Joseph-Vanderpool JR et al (1994). Sleep in fall/winter seasonal affective disorder: effects of light and changing season. J Psychosom Res 38: 323-337.

Avery DH, Dahl K, Savage MV, Brengelmann GL, Larsen LH, Kenny MA et al (1997). Circadian temperature and cortisol rhythms during a constant routine are phase-delayed in hypersomnic winter depression. Biol Psychiatry 41: 1109-1123.

Benca RM, Obermeyer WH, Thisted RA, Gillin JC (1992). Sleep and psychiatric disorders. Arch Gen Psychiatry 49: 651-668.

Bloching B, Dechêne C, Täschner KL (2000). Outlasting antidepressant effect of late partial sleep deprivation by bright light therapy. J Sleep Res 9(Suppl 1): 21.

Borbély AA, Baumann F, Brandeis D, Strauch I, Lehmann D (1981). Sleep deprivation: effect of sleep stages and EEG power density in man. Electroencephalogr Clin Neurophysiol 51: 483-493.

Brunner DP, Kräuchi K, Dijk DJ, Leonhardt G, Haug H-J, WirzJustice A (1996). Sleep EEG in seasonal affective disorder and control women: effects of midday light treatment and sleep deprivation. Biol Psychiatry 40: 485-496.
Cajochen C, Brunner DP, Kräuchi K, Graw P, Wirz-Justice A (1995). Power density in theta/alpha frequencies of the waking EEG progressively increases during sustained wakefulness. Sleep 18: $890-894$.

Cajochen C, Brunner DP, Kräuchi K, Graw P, Wirz-Justice A (2000). EEG and subjective sleepiness during extended wakefulness in seasonal affective disorder: circadian and homeostatic influences. Biol Psychiatry 47: 610-617.

Cajochen C, Khalsa SB, Czeisler CA, Dijk DJ (1998). Time course of EEG power density during wakefulness and subjective sleepiness during a 24-h constant routine. Sleep 21: 243.

Cajochen C, Khalsa SBS, Wyatt JK, Czeisler CA, Dijk DJ (1999). EEG and ocular correlates of circadian melatonin phase and human performance decrements during sleep loss. Am J Physiol 277: R640-R649.

Cajochen C, Wyatt JK, Czeisler CA, Dijk DJ (2002). Separation of circadian and wake duration-dependent modulation of EEG activation during wakefulness. Neuroscience 114: 1047-1060.

Dijk DJ, Beersma DGM, Daan S (1987). EEG power density during nap sleep: reflection of an hourglass measuring the duration of prior wakefulness. J Biol Rhythms 2: 207-219.

Dumont M, Macchi MM, Carrier J, Lafrance C, Hébert M (1999). Time course of narrow frequency bands in the waking EEG during sleep deprivation. NeuroReport 10: 403-407.

Eastman CI, Young MA, Fogg LF, Liu L, Meaden PM (1998). Bright light treatment of winter depression: a placebo-controlled trial. Arch Gen Psychiatry 55: 883-889.

Elmore SK, Dahl K, Avery DH, Savage MV, Brengelmann GL (1993). Body temperature and diurnal type in women with seasonal affective disorder. Health Care Women Int 14: $17-26$.

Finelli LA, Baumann H, Borbély AA, Achermann P (2000). Dual electroencephalogram markers of human sleep homeostasis: correlation between theta activity in waking and slow-wave activity in sleep. Neuroscience 101: 523-529.

Gillberg M, Kecklund G, Åkerstedt T (1994). Relation between performance and subjective ratings of sleepiness during a night awake. Sleep 17: 236-241.

Graw P, Haug H-J, Leonhardt G, Wirz-Justice A (1998). Sleep deprivation response in seasonal affective disorder during a $40-\mathrm{h}$ constant routine. J Affect Disord 48: 69-74.

Hemmeter U, Bischof R, Hatzinger M, Seifritz E, HolsboerTrachsler E (1998). Microsleep during partial sleep deprivation in depression. Biol Psychiatry 43: 829-839.

Koorengevel KM, Beersma DGM, den Boer JA, van den Hoofdakker RH (2002a). Sleep in seasonal affective disorder patients in forced desynchrony: an explorative study. J Sleep Res 11: 347-356.

Koorengevel KM, Beersma DGM, den Boer JA, van den Hoofdakker RH (2002b). A forced desynchrony study of circadian pacemaker characteristics in seasonal affective disorder. $J$ Biol Rhythms 17: 463-475.

Lam RW, Gorman CP, Michalon M, Steiner M, Levitt AJ, Corral MR et al (1995). Multicenter, placebo-controlled study of fluoxetine in seasonal affective disorder. Am J Psychiatry 152: $1765-1770$.

Lam RW, Levitan RD (2000). Pathophysiology of seasonal affective disorder: a review. J Psychiatry Neurosci 25: 469-480.

Lavie P (1986). Ultrashort sleep-waking schedule. III. 'Gates and forbidden zones' for sleep. Electroencephalogr Clin Neurophysiol 63: 414-425.

Lewy AJ, Bauer VK, Ahmed S, Thomas KH, Cutler NL, Singer CM et al (1998a). The human phase response curve (PRC) to melatonin is about 12 hours out of phase with the PRC to light. Chronobiol Int 15: 71-83.

Lewy AJ, Bauer VK, Cutler NL, Sack RL (1998b). Melatonin treatment of winter depression: a pilot study. Psychiatry Res 77: $57-61$. 
Lewy AJ, Kern HA, Rosenthal NE, Wehr TA (1982). Bright artificial light treatment of a manic-depressive patient with a seasonal mood cycle. Am J Psychiatry 139: 1496-1498.

Lewy AJ, Lefler BJ, Hasler BP, Bauer VK, Bernert RA, Emens JS (2003). Plasma DLMO $_{10}$ zeitgeber time 14: the therapeutic window for phase-delayed winter depressives treated with melatonin. Chronobiol Int 20: 1215-1217.

Lewy AJ, Sack RL, Miller LS, Hoban TM (1987). Antidepressant and circadian phase-shifting effect of light. Science 235: 352-354.

Loving RT, Kripke DF, Shuchter SR (2002). Bright light augments antidepressant effects of medication and wake therapy. Depress Anxiety 16: 1-3.

Neumeister A, Goessler R, Lucht M, Kapitany T, Bamas C, Kasper S (1996). Bright light stabilizes the antidepressant effect of sleep deprivation. Biol Psychiatry 39: 16-21.

Palchikov VE, Zolotarev DY, Danilenko KV, Putilov AA (1997). Effects of the seasons and of bright light administered at different times of day on sleep EEG and mood in patients with seasonal affective disorder. Biol Rhythm Res 28: 166-184.

Putilov AA, Danilenko KV, Russkikh GS, Duffy LK (1996). Phase typing of patients with seasonal affective disorder: a test for the phase shift hypothesis. Biol Rhythm Res 27: 431-451.

Putilov AA, Russkikh GS, Danilenko KV (2000). Phase of melatonin rhythm in winter depression. Adv Exp Med Biol 460: 441-458.

Riemann D, Konig A, Hohagen F, Kieman A, Voderholzer U, Backhaus J et al (1999). How to preserve the antidepressant effect of sleep deprivation: a comparison of sleep phase advance and sleep phase delay. Eur Arch Psychiatry Clin Neurosci 249: 231-237.

Rosenthal NE, Genhart M, Sack DA, Skwerer RG, Wehr TA (1987). Seasonal affective disorder and its relevance for the understanding and treatment of bulimia. In: Hudson JJ, Pope Jr HG (eds). The Psychology of Bulimia. American Psychiatric Press: Washington, DC. pp 205-228.

Rosenthal NE, Sack DA, Gillin JC, Lewy AJ, Goodwin FK, Davenport $\mathrm{Y}$ et al (1984). Seasonal affective disorder: a description of the syndrome and preliminary findings with light therapy. Arch Gen Psychiatry 41: 72-80.

Strogatz SH, Kronauer RE, Czeisler CA (1987). Circadian pacemaker interferes with sleep onset at specific times each day: role in insomnia. Am J Physiol 253: R172-R178.

Taillard J, Philip P, Coste O, Sagaspe P, Bioulac B (2003). The circadian and homeostatic modulation of sleep pressure during wakefulness differs between morning and evening chronotypes. J Sleep Res 12: 275-282.

Terman JS, Terman M, Lo ES, Cooper TB (2001). Circadian time of morning light administration and therapeutic response in winter depression. Arch Gen Psychiatry 58: 69-75.

Williams JBW, Link MJ, Rosenthal NE, Terman M (1988). Structured Interview Guide for the Hamilton Depression Rating Scale: Seasonal Affective Disorder Version (SIGH-SAD). New York State Psychiatric Institute: New York.

Wirz-Justice A, Kräuchi K, Brunner DP, Graw P, Haug H-J, Leonhardt G et al (1995). Circadian rhythms and sleep regulation in seasonal affective disorder. Acta Neuropsychiatr 7: $41-43$.

Wirz-Justice A, Van den Hoofdakker RH (1999). Sleep deprivation in depression: What do we know, where do we go? Review. Biol Psychiatry 46: 445-453.

Zaidan R, Geoffriau M, Brun J, Taillard J, Bureau C, Chazot G et al (1994). Melatonin is able to influence its secretion in humans: description of a phase-response curve. Neuroendocrinology 60: 105-112.

Zhdanova IV, Wurtman RJ (1997). Efficacy of melatonin as a sleeppromoting agent. J Biol Rhythms 12: 644-650. 